\title{
Neurosurgical Focus, July 1998
}

\author{
Topic Editors: Keiji Sano, M.D., and Masao Matsutani, M.D. \\ Introduction: Germ Cell Tumors of the Central Nervous System
}

Since the famous comment by Globus and Silbert[2] in 1931 that tumors of the pineal body are a constant challenge to both clinicians and pathologists, the biology and treatment of intracranial germ cell tumors have attracted much attention. Although there are many reports concerning these fields, controversy continues to surround their pathogenesis, their biological behavior, their diagnostic evaluation by neuroimaging, tumor marker studies, and the appropriate treatment for the different histological subtypes.

In this issue of Neurosurgical Focus, the authors provide readers with wide range of recent information concerning the pathogenesis of intracranial germ cell tumors to their multidisciplinary treatment. In the first article, Sano proposes a hypothesis for the pathogenesis of these tumors from the perspective of embryonic development and his clinical experiences.

In examining the different radio- and chemosensitivity between germinomas and nongerminomatous tumors, Nishikawa, et al., compared the expression of p53 and p21 WAF1/Cip 1 proteins by using immunohistochemistry and suggested that the overexpression of $\mathrm{p} 21$ WAF1/Cip1 in nongerminomatous tumors may correlate with decreased sensitivity to treatments. As for the frequent dissemination and multiple occurrence of germ cell tumors, Huang, et al., found that the expression of CD44 adhesion molecules in germinomas may contribute to the tumor cell migration, adhesion on cerebrospinal fluid dissemination, and/or multiple tumor locations. Rogers, et al., observed that the titer of human chorionic gonadotropin-beta in lumbar cerebrospinal fluid is 10 times higher than that in serum and discussed the blood-brain barrier in the pineal gland.

In patients with germinomas, radiation therapy after surgery produced excellent 10-year survival rates of approximately $90 \%$; the remaining $10 \%$ experienced recurrence or dissemination. Murakami, et al., compared pathological composition by means of immunohistochemical analysis of MIB-1 labeling indices and suggested that patients with germinomas with granulomatous inflammation may have a better prognosis than those with ordinary germinomas. Because of the observation made using magnetic resonance imaging that tumors in the suprasellar region originated from the neurohypophysis,[1] the confirmation of this origin by autopsy studies, $[3,4]$ and the finding that they are characteristically accompanied by hypothalamic anterior pituitary dysfunction[5] it is now reasonable to adopt the term "neurohypophyseal tumor" for suprasellar tumors. Kato, et al., treated seven patients with neurohypophyseal germinomas who presented with solely diabetes insipidus alone without a radiologically detectable tumor for months to years. These authors proposed a new concept of occult neurohypophyseal germinomas. 
After the establishment of effective combination chemotherapy with cisplatin for gonadal germ cell tumors, nongerminomatous germ cell tumors in the brain have become candidates for chemotherapy. However, the chemotherapeutic effect has not yet been evaluated in a large study group. Matsutani, et al., reported the preliminary results of a Phase II study in Japan, consisting of a postsurgical chemotherapy and radiation therapy. Their treatment protocol succeeded in achieving an excellent initial response to the combined therapy in germinomas and moderately malignant nongerminomatous tumors.

\section{References}

1. Fujisawa I, Asato R, Okumura R, et al: Magnetic resonance-imaging of neurohypophyseal germinomas. Cancer 68:1009-1014, 1991

2. Globus JH, Silbert S: Pinealomas. Arch Neurol Psychiatry 25:937-985, 1931

3. Jellinger K: Primary intracranial germ cell tumors. Acta Neuropathol 25:291-306, 1973

4. Lewis I, Baxter DW: Atypical teratoma of the pineal. Can Med Assoc J 89:103-110, 1963

5. Sklar CA, Grumbach MM, Kaplan SL, et al: Hormonal and metabolic abnormalities associated with central nervous system germinoma in children and adolescents and the effect of therapy: report of 10 patients. J Clin Endocrinol Metab 52:9-16, 1981

\section{Table of Contents Return to Na//OCB}

